Nonlin. Processes Geophys., 15, 445-455, 2008

www.nonlin-processes-geophys.net/15/445/2008/

(C) Author(s) 2008. This work is distributed under

the Creative Commons Attribution 3.0 License.

\title{
Solar cycle dependence of scaling in solar wind fluctuations
}

\author{
S. C. Chapman, B. Hnat, and K. Kiyani \\ Centre for Fusion, Space and Astrophysics, Physics Department, University of Warwick, Coventry, CV4 7AL, UK
}

Received: 11 March 2008 - Revised: 7 May 2008 - Accepted: 13 May 2008 - Published: 9 June 2008

\begin{abstract}
In this review we collate recent results for the statistical scaling properties of fluctuations in the solar wind with a view to synthesizing two descriptions: that of evolving MHD turbulence and that of a scaling signature of coronal origin that passively propagates with the solar wind. The scenario that emerges is that of coexistent signatures which map onto the well known "two component" picture of solar wind magnetic fluctuations. This highlights the need to consider quantities which track Alfvénic fluctuations, and energy and momentum flux densities to obtain a complete description of solar wind fluctuations.
\end{abstract}

\section{Introduction}

The solar wind provides a unique laboratory for the study of Magnetohydrodynamic (MHD) turbulence with a magnetic Reynolds number estimated to exceed $10^{5}$ (Matthaeus et al., 2005; see also Goldstein and Roberts, 1999). Solar wind monitors such as WIND and ACE provide in situ observations of bulk plasma parameters at $\sim 1 \mathrm{AU}$ in the ecliptic, spanning time intervals from minutes to years. These are complemented by observations over a range of heliographic distances and latitudes, most notably from ULYSSES which has provided several polar passes. These observations suggest turbulence via the statistical properties of their fluctuations (Tu and Marsch; Goldstein, 1995; 2001); a clear scaling range in the magnetic field power spectrum extends from tens of seconds to a few hours with an exponent evolving toward the Kolmogorov (Kolmogorov, 1941, hereafter K41) value of $\sim-5 / 3$; non-Gaussian probability densities of fluctuations (Sorriso-Valvo et al., 1999; Hnat et al., 2002); and intermittency (that is, multifractality seen in the struc-

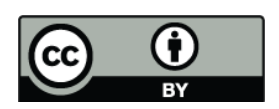

Correspondence to: S. C. Chapman (s.c.chapman@warwick.ac.uk) ture functions). Alfvénic fluctuations dominate the observed power in the solar wind magnetic field with propagation principally away from the sun implying solar origin (e.g. Horbury et al., 2005). The Kolmogorov- like value of the power spectral exponent is therefore somewhat unexpected (e.g. Tu and Marsch, 1995; Goldstein, 2001) from the point of view of incompressible magnetohydrodynamic turbulence; and indeed, intervals can be found where different magnetic field and velocity components simultaneously exhibit scaling consistent with $-5 / 3$ and $-3 / 2$ spectral exponent (e.g. Veltri, 1999). Intermittency modifies the power spectral exponent and this has been suggested to account for the observed $\sim-5 / 3$ power spectra in terms of some incompressible MHD phenomenologies (Carbone et al., 1993), also this scaling can simply be difficult to distinguish in low order moments (Carbone et al., 1995).

Alfvénic fluctuations, when isolated by the use of Elsasser variables (e.g. Horbury et al., 2005) and decomposed by considering different average magnetic field orientations that occur at different times, are found to be multicomponent (Matthaeus et al., 1990) and coupled (Milano et al., 2004). This observationally inspired picture is then of an essentially incompressible, multicomponent Alfvénic turbulence with a significant population of Alfvénic fluctuations that evolve to have wavevectors almost perpendicular to the background magnetic field, leading to a 'fluid- like' phenomenology, and the $-5 / 3$ power spectral slope. Critical to quantifying the scaling properties of fluctuations projected w.r.t. some background magnetic field direction is how the background field is extracted from the data. If the turbulence is sufficiently weak, one might envisage that a macroscopic average (over a typical inertial range upper timescale of an hour, say) is appropriate; however for strong turbulence the relevant background field becomes a function of scale and thus may enter into the resultant scaling exponents. Here we review a recent example (Chapman and Hnat, 2007) which includes the scale dependent local field.

Published by Copernicus Publications on behalf of the European Geosciences Union and the American Geophysical Union. 
Solar wind density fluctuations also show nontrivial scaling (Tu and Marsch, 1995 and more recently Hnat et al., 2003), remote observations suggest that these are not simply proportional to that in magnetic field (Spangler et al., 2004). A topical question is then whether the turbulence is compressible (Hnat et al., 2005): this is important since if it is the case, the evolution of fluctuations in the expanding solar wind cannot be captured by models which describe the observed Alfvénic properties in terms of fluctuating coronal fields that have advected passively in the expanding solar wind (see e.g. Giacalone et al., 2006). The solar wind does also carry with it the remnant signature of heating at the corona. At frequencies below the inertial range, the solar wind exhibits an energy containing range which shows $\sim 1 / f$ scaling (Matthaeus and Goldstein, 1986). Many studies of coronal heating mechanisms are in terms of the scaling properties of coronal structures (Schrijver et al., 1998; Tu et al., 2005), heating rates (Klimchuk and Porter, 1995) and diffusion via random walks of magnetic field lines (Giacalone et al., 2006), all of which suggest self-similar processes. Solar flares show scale invariance in their energy release statistics over several orders of magnitude (Aschwanden, 2000; Schwanden and Parnell, 2002) which has been discussed in terms of Self-Organized Criticality (SOC, see e.g. Lu and Hamilton, 1991; Hughes et al., 2003; Dendy et al., 2007). There is also recent evidence, that we review here, in noncascade quantities, such as magnetic energy density, of a signature within the inertial range that shows scaling that correlates with the level of magnetic complexity in the corona (Hnat et al., 2007; Kiyani et al., 2007).

The purpose of this review is to bring together two quantitative features of solar wind fluctuations seen on timescales that are within the observed inertial range of turbulence. The first of these is anisotropy, and we discuss prospects for quantitative comparison between the predictions of turbulence theories, and the observed scaling exponents. The second of these is a signature of scaling at solar maximum that is self- affine (fractal) and not multifractal to good precision; the suggestion being that this is of coronal origin and is not a signature of turbulence per- se. We will begin with a similarity approach to the intermittency free scaling properties of turbulence. This provides a framework, distinct from probing the intermittency of the flow, that distinguishes quantitatively between turbulence phenomenologies. Importantly, it also highlights that scaling encompasses a wider class of behaviour than turbulence. In the case of the solar wind, this points to the possibility that the observed anisotropic scaling may not be solely the result of in situ turbulence, but may instead be the result of a superposition of signatures that are turbulent, and that are scaling but of direct coronal origin.

Since the above scenarios all predict scaling in the statistics of fluctuations, quantifying this scaling is central to our understanding of the solar wind. Quantifying these fluctuations is also needed to model the transport of solar energetic particles and galactic cosmic rays within the heliosphere.
The measurement of scaling exponents with sufficient precision to distinguish scenarios for coronal heating and the predictions of various MHD turbulence theories presents significant challenges where datasets are finite. We do not present an exhaustive review of techniques here, rather we highlight some recent developments that have yielded new insights into the solar wind.

\section{Scaling exponents, MHD turbulence models and sim- ilarity analysis}

Theories of hydrodynamic turbulence aim to predict the statistical properties of fluctuations in components of velocity by considering ensemble averages. Fluctuations in the velocity field can be characterized by the difference in components, or in the magnitude, $\delta v=v(r+L)-v(r)$ at two points separated by distance $L$. The dependence of $\delta v$ upon $L$ is determined in a statistical sense through the moments $\left\langle\delta v^{p}>\right.$, where $\langle\ldots\rangle$ denotes an ensemble average (practically speaking, assuming ergodicity an average over $r$ ). Statistical theories of turbulence based on details of the phenomenology then aim to predict scaling of the form $<\delta v_{L}^{p}>\sim L^{\zeta(p)}$ with exponents $\zeta(p)$.

It is instructive to organize these ideas in terms of universality (see e.g. Sethna et al., 2001) in as far as it can be identified. We will not present an exhaustive discussion here in the context of hydrodynamic turbulence (see e.g. Frisch, 1995 for a summary) but rather, provide a context within which to order the various results for MHD turbulence. We will apply similarity analysis (Barenblatt, 1996 also known as the Buckingham $\Pi$ theorem Buckingham, 1914) to the problem of MHD turbulence. The central idea of similarity analysis is that if one has the relevant quantities $Q_{1 . . N}$ that describe a given system, then the general solution of the system's behaviour must be a function of the possible dimensionless groups $\Pi_{1 . . M}\left(Q_{1 . . N}\right)$ which can be formed from the $Q_{1 . . N}$. The (unknown) function $F\left(\Pi_{1}, \Pi_{2}, . . \Pi_{M}\right)$ is universal, describing all systems that depend on the $Q_{1 . . N}$ through the $\Pi_{1 . . M}\left(Q_{1 . . N}\right)$ and the relationships between them. Thus this method can lead to information about the solution of a class of systems where the governing equations are unavailable. If we have $N$ quantities expressed in $R$ dimensions (i.e. mass, length, time) then there are $M=N-R$ dimensionless groups. To identify these, we simply tabulate the relevant quantities, the $Q_{1 . . N}$.

We now use similarity analysis to obtain the turbulent energy spectrum. We insist that the system is in steady state so that there is an average energy per unit wavenumber (or per characteristic lengthscale) and an average rate of energy input, which are constants. The relevant quantities are then shown in Table 1.

The first three variables, that is, energy per wavenumber, wavenumber and rate of energy input are the minimum that we can consider and give the scaling behaviour of ideal in- 
compressible hydrodynamic turbulence in the inertial range. We have $N=3$ and $R=2$ so there is only one dimensionless group:

$\Pi_{1}=\frac{E^{3}(k) k^{5}}{\epsilon_{0}^{2}}$

and the universal solution is some $F\left(\Pi_{1}\right)$. The simplest assumption is that $\Pi_{1}$ is a constant, $C$, of order unity, so that rearranging (1) gives:

$E(k)=C \epsilon_{0}^{2 / 3} k^{-5 / 3}$

that is, we recover "Kolmogorov's 5/3" law for hydrodynamic turbulence with a single, universal scaling exponent that is completely constrained by the similarity analysis.

To discuss incompressible MHD, we introduce an additional relevant quantity, the Alfvén speed $v_{0}$. With the above quantities we now have $N=4$ and $R=2$ which gives a second dimensionless group:

$\Pi_{2}=\frac{v_{0}^{2}}{E k}$

To proceed we now need some postulate to relate $\Pi_{1}$ and $\Pi_{2}$. For turbulence, a simple assumption is that the $\Pi_{1}$ and $\Pi_{2}$ are related by some single scaling exponent $\alpha$, that is, in the absence of intermittency, so that $\Pi_{1}=\Pi_{2}^{\alpha}$. This corresponds to an energy spectrum of:

$E(k) \sim k^{-(5+\alpha) /(3+\alpha)}$

with the anomalous scaling exponent $\alpha$ to be determined. Importantly, the above analysis does not specify the nature of the fluctuations, eddies or structures that mediate the cascade (or even, its direction). Thus it captures the scaling of any cascading quantity that carries 'mass normalized' kinetic energy, that is, with physical dimension $[L]^{2}[T]^{-2}$. The energy spectrum $E(k)$ has dimension of (mass normalized) kinetic energy per wavenumber, thus the above also applies to processes that are anisotropic in $k$ provided that the Fourier transform is understood to be taken over the relevant dimension.

The scaling exponent $\alpha$ is anomalous meaning that it is no longer completely constrained by the similarity analysis. Additional constraints introduced by the phenomenology can determine $\alpha$. These include anisotropy (a constraint on the effective Euclidean dimension of the $k$ space), and for weak turbulence, the wave dispersion relation and constraints on allowed wave- wave interactions. It is thus open as to whether MHD turbulence is in this sense universal. Numerical and analytical studies of incompressible MHD then provide different predictions for $\alpha$, depending upon the strength of the turbulence, the strength of the background magnetic field, and anisotropy. Iroshnikov and Kraichnan's (Iroshnikov, 1964; Kraichnan, 1965; hereafter WI) original isotropic, weak (random phase) phenomenology leads to a $\sim k^{-3 / 2}$ spectrum. Introducing anisotropy in the weak
Table 1. Buckingham $\Pi$ theorem applied to homogeneous turbulence (first 3 rows); if there is also a relevant characteristic speed this adds an extra quantity (row 4).

\begin{tabular}{ccl}
\hline Quantity & dimension & description \\
\hline$E(k)$ & {$[L]^{3}[T]^{-2}$} & (mass normalized) average energy \\
& & per unit wavenumber \\
$\epsilon_{0}$ & {$[L]^{2}[T]^{-3}$} & rate of energy input \\
$k$ & {$[L]^{-1}$} & wavenumber \\
$v_{0}$ & {$[L][T]^{-1}$} & characteristic speed \\
\hline
\end{tabular}

case leads to $\mathrm{a} \sim k_{\perp}^{-2}$ spectrum for fluctuations perpendicular to the background magnetic field (e.g. Galtier et al., 2000; hereafter WA). In contrast, strong turbulence phenomenology (hereafter SA see Goldreich and Sridhar, 1997 and references therein) yields a $\sim k_{\perp}^{-5 / 3}$ spectrum. This symmetric case where the fluxes of oppositely directed Alfvén waves are equal does not however strictly apply to the solar wind (Goldreich and Sridhar, 1997), where the fluxes are observed to be asymmetric. Recent numerical simulations (Müller and Grappin, 2005), and analysis (Boldyrev, 2006 hereafter SB) obtain a $\sim k_{\perp}^{-3 / 2}$ spectrum for the case of a strong local background magnetic field. This $-3 / 2$ exponent, combined with the anisotropy of the fluctuations, is in contradiction with WI (either isotropic, $-3 / 2$ exponent or anisotropic, -2 exponent) and SA (anisotropic, -5/3 exponent) phenomenologies. In summary, then, in the absence of intermittency, these phenomenologies give $\alpha=1$ (WI), $\alpha_{\perp}=0$ (SA), $\alpha_{\perp}=1$ (SB) and $\alpha_{\perp}=-1$ (WA).

These models, although usually expressed in terms of the energy spectrum, predict the intermittency free scaling exponent that applies to all the moments of the differences, i.e. for hydrodynamic turbulence, the $<\delta v_{L}^{p}>\sim L^{\alpha p}$.

Intermittency, when expressed as scale dependence of the energy transfer rate $\epsilon_{L}$, enters via the scaling properties of the differences. Intermittency in the context of MHD turbulence has been studied numerically (see e.g. Müller and Biskamp, 2000 also Merrifield et al., 2006, 2007) with reference to models such as that of She and Leveque (She and Leveque, 1994; see also Politano and Pouquet, 1995). Dimensional analysis, as we shall see next, provides an overall framework from which we can obtain intermittency free exponents that are independent of this phenomenology. We will phrase this discussion in terms of velocity differences, however as above this dimensional analysis will apply to any quantity that carries "mass normalized" kinetic energy, with physical dimension $[L]^{2}[T]^{-2}$. Again, consider a cascade (direction unspecified) in which fluctuation $\delta v$ on lengthscale $L$ transfers kinetic energy $\delta v^{2}$ on timescale $T \sim L / \delta v$, implying an energy transfer rate $\epsilon_{L} \sim \delta v^{2} / T \sim \delta v^{3} / L$. If the statistics of the fluctuations in the energy transfer rate are 
independent of $L$, its $p$ moments will be $\left\langle\epsilon_{L}^{p}>\sim \epsilon_{0}^{p}\right.$ where the constant $\epsilon_{0}$ is the average rate of energy transfer. This leads to the K-41 scaling $<\delta v_{L}^{p}>\sim L^{p / 3}$. For incompressible MHD turbulence, we again consider an additional relevant quantity which will introduce an additional factor here that must be dimensionless (Barenblatt, 1996). To obtain an expression solely in terms of velocity differences we thus introduce a characteristic speed (the Alfvén speed) $v_{0}$ into the problem, if instead we were considering magnetic field fluctuations we could instead introduce the constant background field strength $B_{0}$. This simply modifies the energy transfer time to $T \sim(L / \delta v)\left(v_{0} / \delta v\right)^{\alpha}$, leading to an energy transfer rate $\epsilon_{L} \sim \delta v^{(3+\alpha)} / L$ so that $\left\langle\delta v_{L}^{p}>\sim L^{\zeta(p)}\right.$ and

$\zeta(p)=\frac{p}{(3+\alpha)}$

again, with anomalous scaling exponent $\alpha$ to be determined from the phenomenology. This corresponds directly to the intermittency free power spectrum above, since dimensionally, $E(k) \sim<\delta v^{2}>/ k$ giving $E(k) \sim k^{-(5+\alpha) /(3+\alpha)}$.

Deviation from this single exponent (fractal) scaling, that is, intermittency (e.g. Sornette, 2004), can now be introduced through a lengthscale dependence of the fluctuations in energy transfer rate so that $\left\langle\epsilon_{L}^{p}>\sim \epsilon_{0}^{p}\left(L / L_{0}\right)^{\mu(p)}\right.$, where $L_{0}$ is some characteristic lengthscale and $\mu(p)$ is the intermittency correction. The scaling for the moments then becomes:

$\zeta(p)=\frac{p}{(3+\alpha)}+\mu\left(\frac{p}{3+\alpha}\right)$

That this scaling will apply to any cascading quantity $\varepsilon_{c}$ can be seen if we replace $\delta v$ by $\delta \varepsilon_{c}^{1 / 2}$ in the above, where $\varepsilon_{c}$ has dimension $[L]^{2}[T]^{-2}$ or (mass normalized) energy, in the sense that $<\delta \varepsilon_{c}^{p}>\sim L^{\zeta(2 p)}$. This scaling property is obtained without referring to specific phenomenology, arising solely from similarity analysis (Barenblatt, 1996). This does not determine the functional form of $\mu$ or the direction of the cascade for which phenomenology, or governing equations, are needed. For K-41 hydrodynamic turbulence for we have the " $4 / 5$ " law $<\delta v_{L}^{3}>\sim-4 / 5 L$ (e.g. Frisch, 1995) determined from the Navier- Stokes equations (for the equivalent relation for isotropic MHD see Politano and Pouquet, 1998). This follows since $<\delta v^{3}>/ L$ is the energy transfer rate.

The experimental study of the fundamentals of turbulence then centres around measurement of the $\zeta(p)$. A full description requires both the anomalous exponent $\alpha$ and the (difficult to determine) intermittency correction, the $\mu(p)$. However, if the system is in a homogeneous steady state, the average energy transfer rate is uniform so that $\left\langle\epsilon_{L}\right\rangle=\epsilon_{0}$ and $\mu(1)=0$ so that for MHD flows, this simple dimensional argument implies that there is an "intermittency free" exponent (e.g. Chapman and Hnat, 2007):

$\zeta(3+\alpha)=1$ which is independent of the intermittency of the flow. Since the lower order moments are more precisely determined, this offers the possibility to distinguish $\alpha=0$ and $\alpha=1$ for in- situ observations of the solar wind and we give an example in Sect. 5.

Finally, in an infinite domain, homogeneous system, these exponents ideally make direct contact with the class of stable distributions (i.e. Gaussian, Lèvy, and log- Normal; Sornette, 2004). Experimentally (or observationally) one finds that the turbulent fluctuations are large but finite range, and the Probability Density Function (PDF) of fluctuations is non Gaussian, with tails that can be approximated by a stretched exponential or by a power law with exponential truncation (Sorriso-Valvo et al., 1999; Hnat et al., 2003). At least for fractal (selfsimilar) scaling, the scaling exponents can quite generally specify the PDF through, for example, nonlinear Fokker - Planck equations (Hnat et al., 2003; Chapman et al., 2005; Hnat et al., 2005).

\section{Structure function analysis}

We now consider time series from a single spacecraft so that the ensemble averages will be over time rather than over space, the spatial separation above being replaced by a time interval $\tau$ using the Taylor hypothesis (Taylor, 1938; see also Matthaeus et al., 2005). Consistent with almost all experimental studies of turbulence we consider generalized structure functions of a given quantity $x$ :

$S_{p}(\tau)=<|x(t+\tau)-x(t)|^{p}>=<|y(t, \tau)|^{p}>$

on all available time intervals $\tau$ where $<\ldots>$ indicate ensemble averaging over $t$. This statistical scaling with $\tau$ follows that of the modulus moments of the PDF of $y, P(y, \tau)$ :

$S^{p}(\tau)=\left\langle|y|^{p}\right\rangle=\int_{-\infty}^{\infty}|y|^{m} P(y, \tau) d y$

For the case considered here, where a single set of exponents $\zeta(p)$ apply to both the positive and negative fluctuations, the scaling property $S_{p} \sim \tau^{\zeta(p)}$ is preserved under the modulus operation, however the signed moments (as in the sign of the 4/5 law) are not. The odd order moments vanish for a symmetric PDF so that practically, for a finite sample and in the presence of observational uncertainties, unless the PDF is strongly asymmetric they can be poorly determined; hence the modulus moments provide a more robust estimator of the $\zeta(p)$.

A special case that we will test for here is statistical selfsimilarity (fractality) which implies that any PDF at scale $\tau$ can be collapsed onto a unique single variable $\operatorname{PDF} \mathcal{P}_{S}$ :

$P(y, \tau)=\tau^{-H} \mathcal{P}_{s}\left(\tau^{-H} y\right)$,

where $H$ is the Hurst exponent. Equation (10) implies that the increments $y$ are self-affine i.e. they obey the statistical scaling equality 


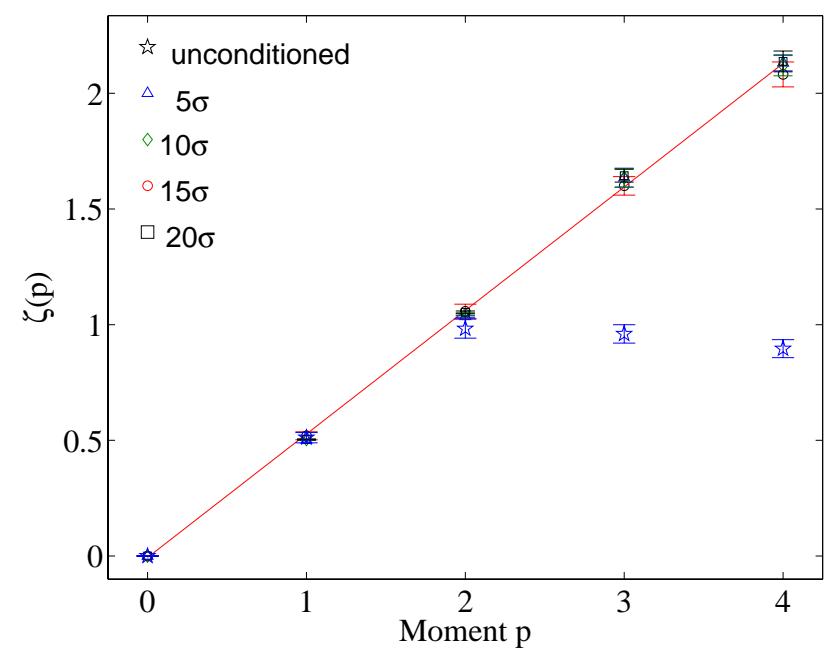

Fig. 1. The effect of conditioning a Levy flight. $\star$ are the $\zeta(p)$ obtained from the raw time series, all other symbols refer to conditioned time series for different values of the limits $y_{ \pm}=A \sigma(\tau)$ where $A=[5-20]$. The conditioned results yield a scaling exponent $H=0.544$ which corresponds to a Lévy index of $\mu=1.84$ (after Chapman et al., 2005).

$y(b \tau) \doteq b^{H} y(\tau)$,

such that the structure functions will scale with $\tau$ as

$S^{p}(\tau)=\tau^{H p} \mathcal{S}_{S}^{p}(1)$

The procedure for extracting the scaling exponents from the data is in principle simply to plot the log structure functions versus $\log (\tau)$, the gradients yield the corresponding scaling exponents, the $\zeta(p)$ for the region where $S_{p} \sim \tau^{\zeta(p)}$. However, finite experimental data sets include a small number of extreme events which have poor representation statistically and may obscure the scaling properties of the time series. One method (Veltri 1999; Chapman et al., 2005, for other approaches see e.g. Katul et al., 1994; Horbury and Balogh, 1997; Kiyani et al., 2006) for excluding these rare events is to fix (large) upper limits $y_{ \pm}$on the magnitude of fluctuations used in computing the structure functions. Importantly, the $y_{ \pm}$must possess the same dependence on the temporal scale $\tau$ as the fluctuation PDF; generally this is not known a priori. A practical approach is simply to base the limit on the standard deviation $\sigma(\tau)$; that is, we choose an upper limit of $y_{ \pm}=A \sigma(\tau)$ where $A=[5-20]$, this matches exactly the scaling of the PDF for the special case of fractal scaling, since the single exponent which rescales $\sigma$ also rescales the entire PDF. We illustrate this in Fig. 1 with a manifestly self-similar process, an $\alpha$-stable Lévy process of index $\mu=1.8(\mu=1 / H$ as in the notation here).

This match of the $y_{ \pm}$scaling to that of the PDF as a whole is only approximate for multifractal timeseries however practically it can still give good convergence (that is, exponents
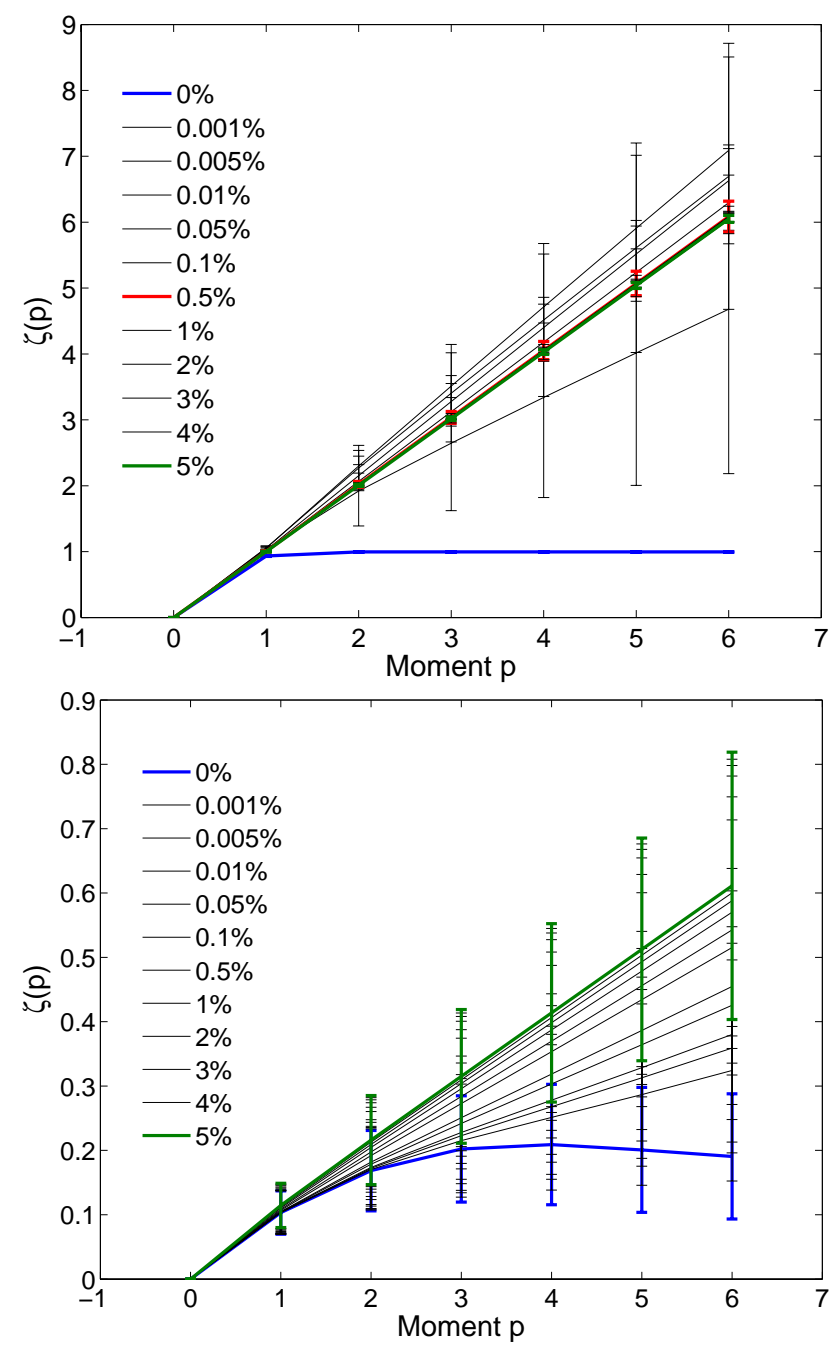

Fig. 2. $\zeta(p)$ Vs. $p$ plots for (a) $\mu=1.0$ symmetric Lévy process and (b) $p=0.6 p$-model process, as outliers are successively removed (after Kiyani et al., 2007).

that are insensitive to the choice of $A$ ). We check for convergence, i.e. that the scaling exponents are not strongly sensitive to the value of the upper limit and are thus reliable. Above $10 \sigma(\tau)$ this process eliminates less than $\sim 1 \%$ of the data considered here.

A procedure that does not impose a priori assumptions on the $y_{ \pm}$was recently proposed by Kiyani et al. (2006) (see also Kiyani et al., 2007). One systematically excludes a minimal percentage of the outlying events $y$ from the integral in (9) so that the statistics of the PDF tails become well sampled. This is done by successively removing (one at a time) the largest outliers from the set of differences $y(t, \tau)$ and recalculating the structure functions $<|y(t, \tau)|^{p}>$ and the corresponding exponents, the $\zeta(p)$. Since the PDF of $y(t, \tau)$ may be asymmetric, outliers are removed successively from both positive and negative tails. For a strongly non- Gaus- 

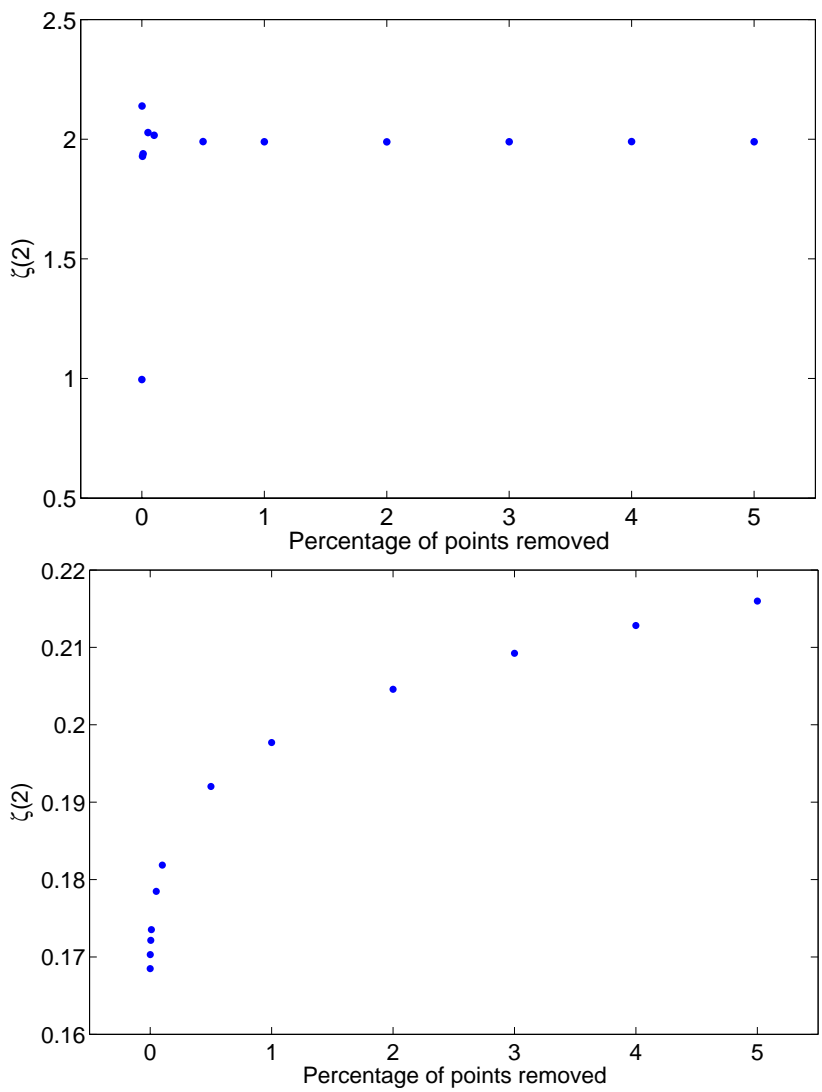

Fig. 3. Exponent of the second moment $\zeta(2)$ vs. the percentage of points excluded for (a) the Lévy model of Fig. 2 and (b) $p$-model.

sian PDF one then should find that if the statistics of the PDF tails are not well sampled, the determined $\zeta(p)$ will vary quite strongly as we successively remove individual outliers (in other words, an individual $y$ data point has a large influence on the value of $\zeta(p)$ ). On the other hand, if the PDF is well sampled statistically, removing a single outlying data point does not strongly influence the resulting value of $\zeta(p)$. If and only if the PDF is well sampled in this sense for a large range of $y$ that extends well into the tails of the distribution then this value of $\zeta(p)$ will provide a good estimate of the scaling behaviour of the $y$. This method is also particularly sensitive in distinguishing self-affine scaling from weak multifractality. Both these points are illustrated in Figs. 2 and 3. In Fig. 2 we compare an $\alpha$-stable Lévy process of index $\mu=1.0$ with a process that is manifestly multifractal, that is, a $p$-model with $p=0.6$. The exponents $\zeta(p)$ have been recomputed as outlying data points are successively removed, and we can see that removing a small fraction, $\sim 0.001 \%$ of the data leads to a large change in the computed $\zeta(p)$. A reliable estimate of the exponents from the data requires rapid convergence to robust values; shown in Kiyani et al. (2006) to be a property of self-affine timeseries. We can see this behaviour in the Lévy model which quickly converges to linear dependence of $\zeta(p)$ with $p$ as expected. The multifractal $p$ model approaches linearity more slowly and is roughly linear after $\sim 3 \%$ of the data is excluded.

We can most clearly see this behaviour if we plot the value of one of the exponents from Fig. 2 versus the percentage of points removed. This is shown for $\zeta(2)$ for the Lévy process (upper panel) and the $p$-model (lower panel) in Fig. 3. As we successively exclude outlying data points, the self-affine Lévy process quickly reaches a constant value for $\zeta(2)=2 / \mu=2.0$ whereas for the multifractal, the $\zeta(2)$ exponent shows a continuing secular drift. Importantly, successively removing outlying data points does not convert the multifractal $p$-model timeseries into a self-affine process. In addition, a plot of $\zeta(p)$ versus $p$ is not sufficient to distinguish self-affine from multifractal behaviour, one also needs to examine the convergence properties of the exponents as in Fig. 3.

\section{The datasets}

The WIND and ACE spacecraft spend extended intervals at $\sim 1$ AU in the ecliptic and provide in-situ magnetic field observations of the solar wind over extended periods covering all phases of the solar cycle. We focus on two studies here. The first study uses $64 \mathrm{~s}$ averaged plasma parameters from ACE for the interval 1 January 1998-31 December 2001, this consists of $\sim 1.6 \times 10^{6}$ samples and is dominated by slow solar wind. The second uses WIND 60 seconds averaged MFI data at the solar maximum year of 2000 and at the solar minimum year of 1996 and ACE $64 \mathrm{~s}$ averaged MFI data for the year 2000 , each consisting of $\sim 4.5 \times 10^{5}$ points. All of the above intervals show a $\sim-5 / 3$ power law scaling inertial range in the power spectra of $|B|$ over several decades and the timescales of fluctuations that we study here are within this inertial range.

\section{Components of velocity fluctuations}

The scaling arguments above encompass the possible realizations of MHD turbulence, a subset of which predict distinct values of the intermittency free scaling exponent $\alpha$. We first verify that for the interval under study, there is a stable region of Alfvénicity that corresponds to the inertial range. This can be seen in Fig. 4 where we plot a measure of the Alfvénicity, that is power spectral density of the difference of forward (+) and backward (-) directed Elsasser variable (see e.g. Horbury et al., 2005) for GSE $x, y, z$ coordinates. In the inertial range (and the range of timescales $\tau$ that we consider here) these ratios are all frequency (that is, $\tau$ ) independent, giving strong fluctuations at timescales above a few hours, the timescale for large scale coherent structures.

Anticipating anisotropic phenomenology, we will now consider vector velocity fluctuations which we will decompose w.r.t. the direction of the magnetic field. In the tur- 


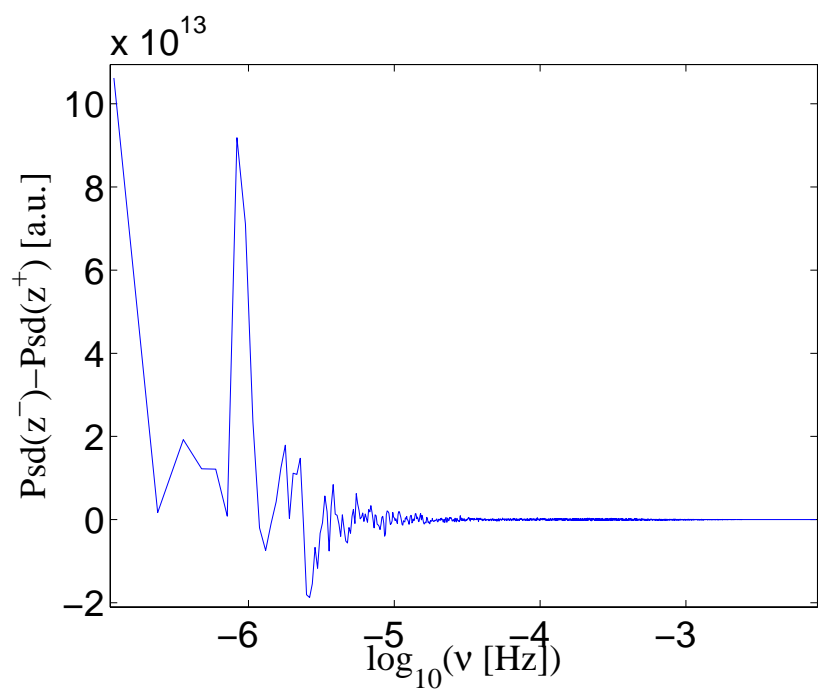

Fig. 4. Aflvénicity of the solar wind interval studied here, defined as the difference of the power spectrum densities of Elsasser variables $z^{-}$and $z^{+}$. Positive values indicate dominant anti-Sunward waves. Data set has been averaged on $1 \mathrm{~h}$ temporal scale.

bulent flow, the magnetic field also fluctuates, but we can consider a local background value by constructing a running average of the vector magnetic field over the timescale $\tau^{\prime}$. For each interval over which we obtain a difference in velocity $\delta \boldsymbol{v}=\boldsymbol{v}(t+\tau)-\boldsymbol{v}(t)$ we also obtain a vector average for the magnetic field direction $\hat{\boldsymbol{b}}=\overline{\boldsymbol{B}} /|\overline{\boldsymbol{B}}|$ from a vector sum of all the observed vector values between $t-\tau$ and $t+\tau, \overline{\boldsymbol{B}}(t, \tau)=\boldsymbol{B}(t-\tau)+\ldots+\boldsymbol{B}(t+\tau)$. We choose this interval as the minimum (Nyquist) necessary to capture wavelike fluctuations. Velocity differences $\delta \boldsymbol{v}$ which are Alfvénic in character will then have the property that the scalar product $\delta \boldsymbol{v} \cdot \hat{\boldsymbol{b}}$ will vanish. This condition filters out all those fluctuations which generate a velocity displacement perpendicular to the local magnetic field, and is thus less restrictive than the Elsasser (Horbury et al., 2005) variables which select propagating pure Alfvén waves, thus potentially encompassing both weak and strong turbulence phenomenologies.

In Fig. 5 we compare the exponents of $\delta v_{\|}=\delta \boldsymbol{v} \cdot \hat{\boldsymbol{b}}$ with those of $\delta v_{\perp}=\left(\delta \boldsymbol{v} \cdot \delta \boldsymbol{v}-(\delta \boldsymbol{v} \cdot \hat{\boldsymbol{b}})^{2}\right)^{1 / 2}$. These exponents were obtained by conditioning at $A=10$ (that is, removing fluctuations larger than at $10 \sigma(\tau)$ ); we have verified that the exponents are rather weakly sensitive to conditioning provided that $A$ is large Chapman and Hnat (2007). We can see that both these quantities show a clear scaling range with scaling exponents $\zeta(3)$ and $\zeta(4)$ close to unity for $\delta v_{\|}$and $\delta v_{\perp}$ respectively. Thus for a turbulent signal, in the sense of our dimensional analysis above, we obtain, from $\zeta(3+\alpha)=1$, that the intermittency free anomalous scaling exponents are $\alpha_{\|}=0$ and $\alpha_{\perp}=1$. From the figure we can also see that the scaling in $\delta v_{\perp}$ is multifractal, whereas that in $\delta v_{\|}$is closer to self- affine. This permits at least two possibilities: (i) that all of this scaling can be accounted for within turbulence phe-

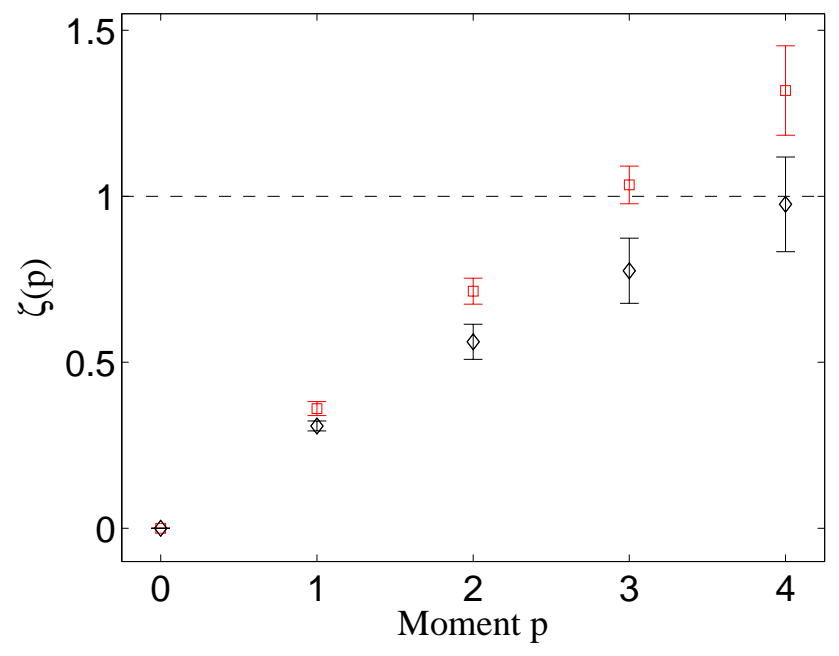

Fig. 5. Scaling exponents $\zeta(p)$ versus $p$ for the structure functions of $\left\langle|\delta \boldsymbol{v} \cdot \hat{\boldsymbol{b}}|^{p}\right\rangle(\square)$ and of the remaining signal $(\diamond)$. Note that $\zeta(3) \approx 1$ and $\zeta(4) \approx 1$ respectively for these quantities.

nomenology or (ii) that one of the components, $\delta v_{\perp}$ say, is due to anisotropic turbulence (and consistent with SB phenomenology) whereas the other, $\delta v_{\|}$, arises from another process. With this in mind, we next review recent evidence of scaling that may have a non- local source, namely the corona.

\section{Fractal signature of the coronal driver}

To discern possible signatures that are of coronal origin, we next consider intervals of data from different phases of the solar cycle. We consider a quantity, magnetic field energy density, which is not anticipated to strongly reflect the Alfvénic fluctuations in the data and is therefore more sensitively dependent on processes other than Alfvénic turbulence. The effect of iterative conditioning is shown in Fig. 6, for magnetic field energy density solar maximum, $B_{\text {Max }}^{2}$ and minimum, $B_{\text {Min }}^{2}$, respectively. We plot $\zeta(p)$ versus $p$ as we successively remove outlying data. In Fig. 6 we see that the $\zeta(p)$ from the raw data move toward a constant level as $p$ increases. This saturation is especially pronounced during solar maximum for the moments of order $p>2$, reminiscent of the behaviour found in Lévy flights (in both standard and fractional cases, e.g. (Watkins et al., 2005), see also (Kiyani et al., 2006)). Different symbols denote the fraction of outliers $K \in[0 \%, 5 \%]$ successively excluded. A straight line has been fitted to pass through $\zeta(p)=0-2$ on both plots. We see that at $K=0.1 \%$ the exponents all approach this line for solar maximum, consistent with a fractal time series. At minimum there is a departure from linear $\zeta(p)$ behaviour which is weak but just resolvable within the errors at higher $p$, even when $K$ is relatively large. The rate of convergence can also be seen to qualitatively differ and is more rapid for solar maximum. 

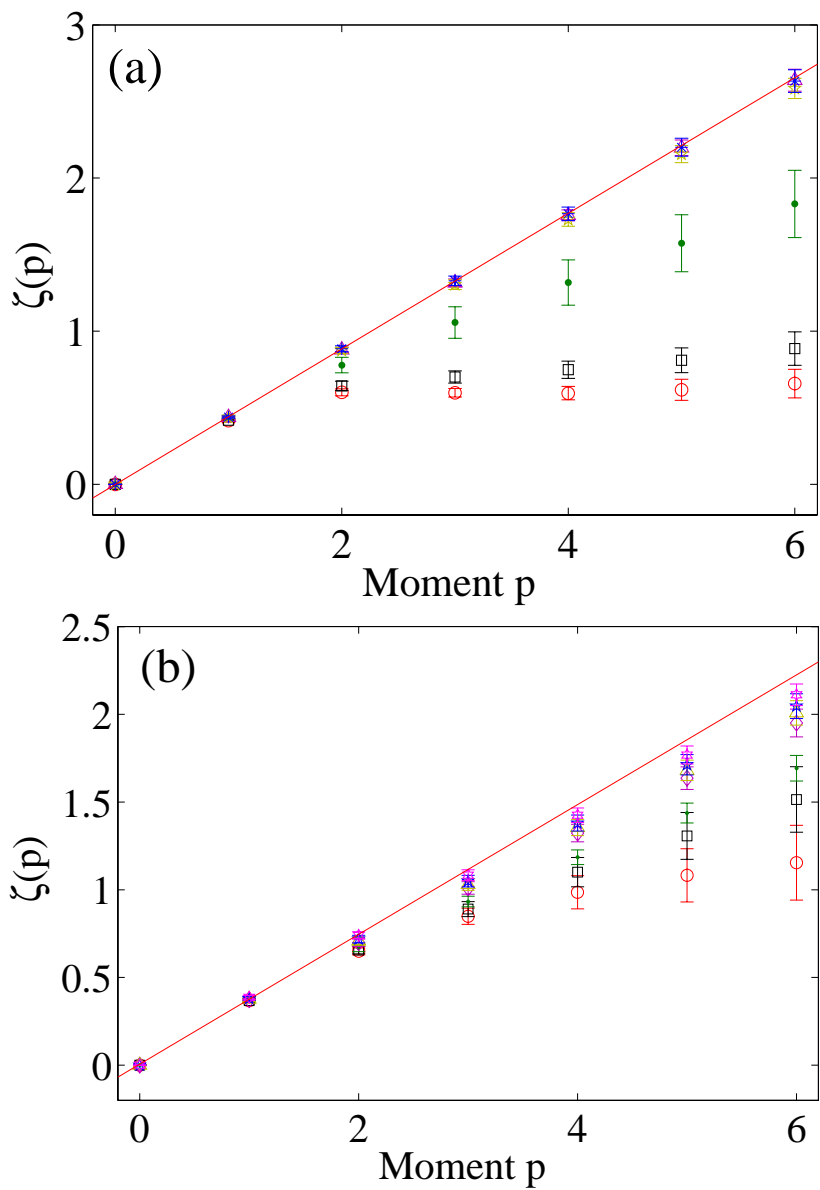

Fig. 6. Scaling exponents $\zeta(p)$ as functions of the order $p$ for (a) solar maximum and (b) solar minimum. Different symbols correspond to the fraction $K$ of excluded points used in conditioning: $\circ-K=0, \square-K=0.005 \%, \bullet-K=0.05 \%, \diamond-K=0.5 \%$, $\triangle-K=1 \%, *-K=2 \%, \star-K=5 \%$. Straight lines have been fitted to pass through $\zeta(p)=0-2$ (after Hnat et al., 2007).

In Fig. 7 we plot $\zeta(2)$ versus the percentage of points removed in $B^{2}$ for the intervals at solar maximum and minimum. The $\zeta$ values for these plots were obtained from an identified scaling range which spanned from $\sim 5.2 \mathrm{~min}-$ utes to $\sim 2.7$ hours (see e.g. Hnat et al., 2003). Comparison of these plots with Fig. 3 here strongly suggests that at solar maximum, the magnetic energy density is self-affine; we can clearly identify a plateau with a $H=\zeta(2) / 2$ value of $H \simeq 0.44 \pm 0.02$ for WIND and $H \simeq 0.45 \pm 0.01$ for ACE. At solar minimum, there is no clear plateau and the behaviour is reminiscent of the multifractal $p$-model. We have thus differentiated the distinct scaling behaviour at solar maximum and solar minimum. Intriguingly, it is at solar maximum that we see self-similar behaviour; whereas at solar minimum the timeseries resembles a multifractal, reminiscent of intermittent turbulence. Since the corona is complex and highly structured at solar maximum, this is highly suggestive that
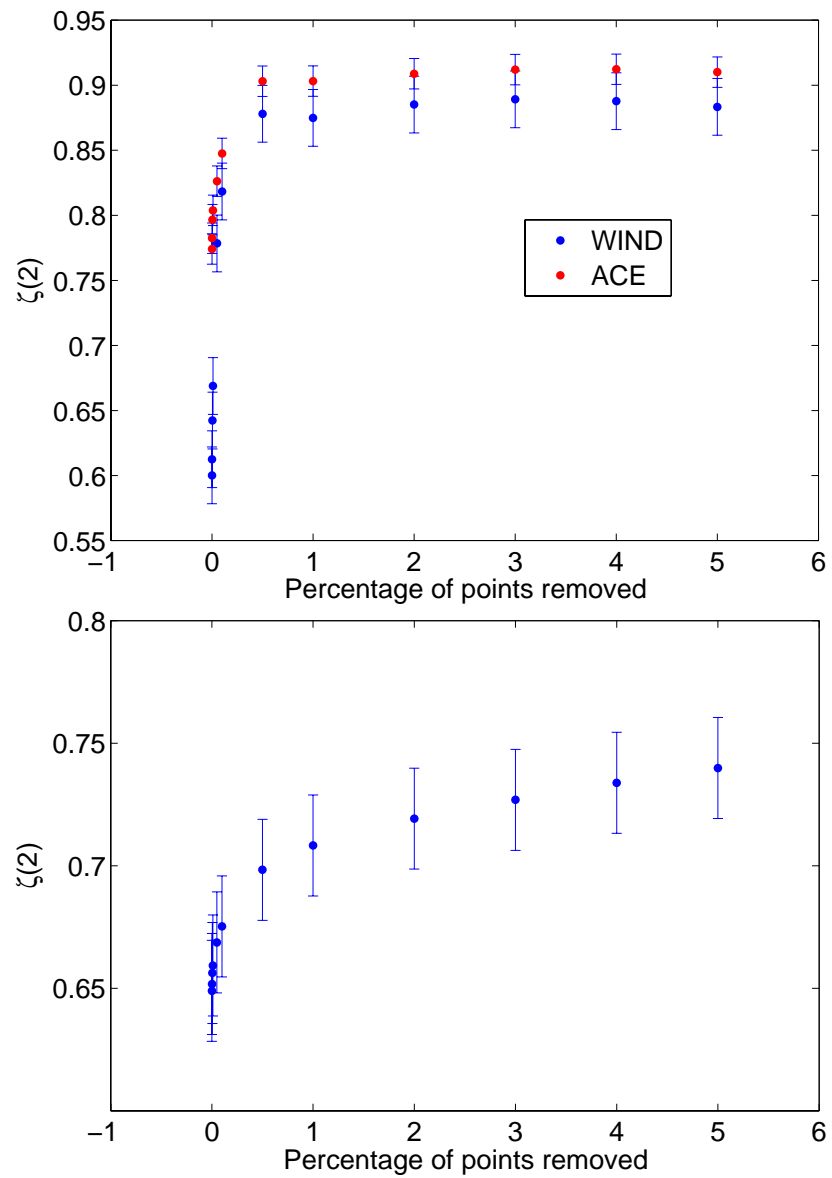

Fig. 7. Exponent of the second moment $\zeta(2)$ vs. the percentage of points excluded for (a) WIND and ACE at solar maximum and (b) WIND at solar minimum (after Kiyani et al., 2007).

this self-similar signature in $B^{2}$ is related to coronal structure and dynamics rather than to local turbulence. We emphasize that this signature appears at temporal scales within the inertial range of the turbulent solar wind.

From the above we would anticipate that the PDF of fluctuations in $B^{2}$ at solar maximum and minimum will not share the same functional form. We verify this in Fig. 8 where we compare the distributions of positive fluctuations in $B_{\mathrm{Min}}^{2}$ and $B_{\mathrm{Max}}^{2}$, at $\tau \approx 30 \mathrm{~min}$, normalized to their respective standard deviations (this log-log plot emphasizes the PDF tails). We see that the PDFs do differ significantly for the entire range of fluctuations. The fluctuations in $B^{2}$ at solar maximum are in many aspects similar to those seen in Lévy flights. A power law tail is evident in the figure at solar maximum over about $1-1.5$ decades in fluctuations of $B^{2}$. This is consistent with, but not unique to, the limiting form of a Lévy PDF, $P_{L}(|\delta x| \rightarrow \infty) \propto|\delta x|^{-(1+\mu)}$. The slope of the best fitted line suggests a $\mu$ value of $\approx 1.4$. At solar minimum, the tails of the PDF suggest an exponential roll-off. 


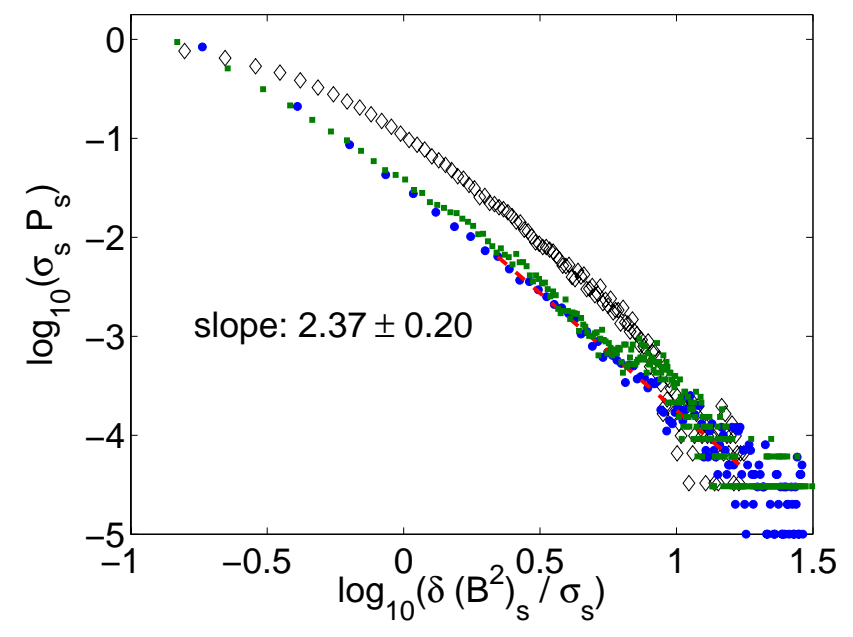

Fig. 8. Tails of the distribution for positive fluctuations in $B^{2}$ : $\diamond$ - WIND at solar minimum, $\bullet-$ WIND at solar maximum and $\square-$ ACE at solar maximum (after Hnat et al., 2007).

The apparent self-similarity of fluctuations at solar maximum suggest that a good model for the timeseries may be a (non- Gaussian) random fractal. In Fig. 9 we have overplotted on the PDF of the data that of several candidate models. A nonlinear Fokker-Planck (F-P) model (Hnat et al., 2003; Chapman et al., 2005) has been developed to describe selfsimilar fluctuations in this context, and is shown on the plot as a dashed line. In the same figure we overplot as a dotted line a Lévy PDF calculated from the characteristic function as $P_{L}(x)=\int d k e^{-i k x} e^{-\gamma|k|^{\mu}}$ with $\mu=1.4$ and $\gamma=0.3$. We can see that both these functions adequately describe the data.

\section{Conclusions}

In this review we have highlighted the distinction between two classes of departure of scaling from that of Kolmogorov's 1941 theory of hydrodynamic turbulence, which predicts for example the classical " $-5 / 3$ " power law power spectrum, and fractal scaling. The first of these, in our terminology, is a single anomalous scaling exponent $\alpha$ which we demonstrate arises straightforwardly from dimensional analysis if we simply insist that there is additional relevant physics in the problem. This exponent $\alpha$ is anomalous in the sense that it is not determined by dimensional analysis alone; different phenomenologies of MHD turbulence predict distinct values of $\alpha$ (and introducing anisotropy leads to a distinct $\alpha_{\|}$and $\alpha_{\perp}$ ). The second of these is due to intermittency of the dissipation; that is, a scale dependence in the energy transfer rate. The assumption of steady state on the average then leads to an "intermittency free" scaling exponent which depends on $\alpha$ but not on the intermittency correction. Since

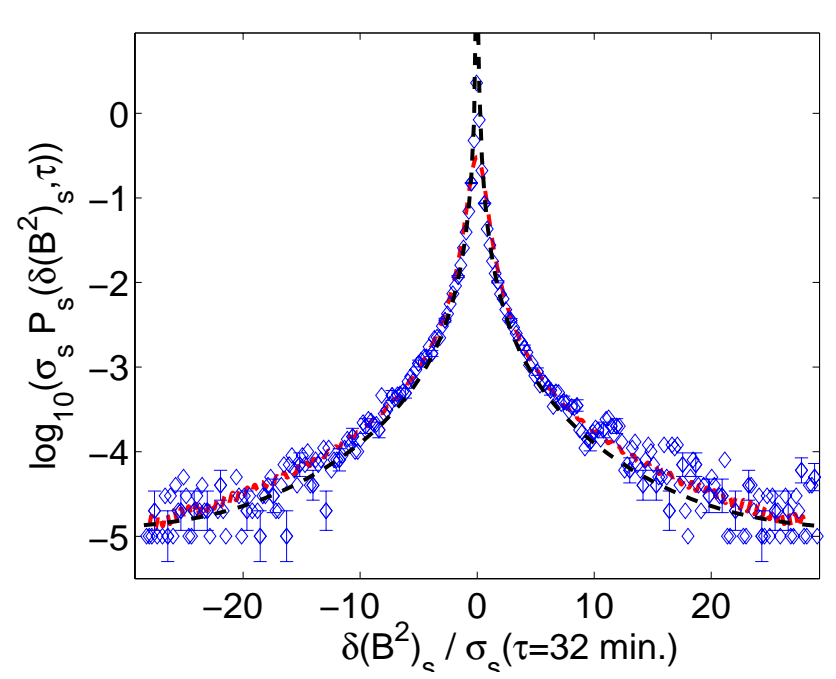

Fig. 9. Fitted curves over observed pdf for $\tau=32$ minutes. Dashed line indicates a Fokker-Planck solution and a dotted line corresponds to a Lévy PDF with $\mu=1.4$ and $\gamma=0.3$.

this is associated with a low order moment this offers a practical possibility to determine $\alpha$ experimentally.

This idea has been tested on solar wind velocity fluctuations in the inertial range which have been decomposed into components parallel to and perpendicular to the local background magnetic field direction (Chapman and Hnat, 2007). The perpendicular velocity component then gives $\alpha_{\perp} \sim 1$ consistent with recent predictions for anisotropic Alfvénic turbulence in a background field. The parallel velocity component gives $\alpha_{\|} \sim 0$ with roughly "K-41-like" scaling. This suggests an important insight into the previously proposed multicomponent nature of solar wind turbulence in that it suggests one of two scenarios. One is that the two components both arise from anisotropic compressible MHD turbulence in the presence of a background field, in which case this determination of their scaling properties points to potential development of theories of MHD turbulence. The other is that the turbulent solar wind is comprised of two weakly interacting components- one (seen in $\delta v_{\perp}$ ) that evolves in the high Reynolds number flow, and the other (seen in $\delta v_{\|}$) from the process that generates the solar wind at the corona. With this in mind, we also have highlighted here evidence for a scaling signature of coronal origin within the inertial range of solar wind turbulence (Kiyani et al., 2007). This relies on a novel technique, that of iteratively conditioning the structure functions (Kiyani et al., 2006), which is a particularly sensitive discriminator of fractality, and where the time series is indeed fractal, quantifies the scaling exponent to good precision. At solar maximum, the timeseries of magnetic energy density is found to be fractal, whereas there is a clearly discernable departure from fractality at solar minimum. This difference is reflected in the functional form of their PDFs and intriguingly, at solar maximum, the PDFs show power law tails. 
The appearance of fractal scaling tracks the increased complexity of the magnetic field structure in the corona at solar maximum. This signature of fractal scaling is found within the inertial range of turbulence seen in the solar wind, that is, coincident with the signature of approximately $-5 / 3$ power law power spectra, rather than at the lower frequencies typically associated with the $1 / f$ scaling seen in energy containing scales. This result may inform understanding of the interplay between the signature of coronal heating and solar wind acceleration, propagated to $1 \mathrm{AU}$ (Milovanov and $\mathrm{Ze}-$ lenyi, 1998), and that of locally evolving turbulence. It highlights the need for careful quantitative determination of exponents both of quantities which track Alfvénic fluctuations, and energy and momentum flux densities to obtain a complete description of solar wind fluctuations. How this relates to the solar cycle dependence of scaling seen in geomagnetic indices (Hnat et al., 2005) is also an open question.

Acknowledgements. The authors thank G. Rowlands for discussions, the WIND and ACE teams for data provision and the STFC for support.

Edited by: A. C. L. Chian

Reviewed by: two anonymous referees

\section{References}

Aschwanden, M. J.: Time variability of the "quiet" sun observed with TRACE. II. Physical parameters, temperature evolution, and energetics of extreme-ultraviolet nanoflares Astrophys. J., 535, 1047, 2000.

Aschwanden, M. J. and Parnell, C. E.: Nanoflare statistics from first principles: fractal geometry and temperature synthesis, Astrophys. J., 572, 1048, 2002.

Barenblatt, G. I.: Scaling, self-similarity, and intermediate asymptotics, CUP, 1996.

Buckingham, E.: On Physically Similar Systems; Illustrations of the Use of Dimensional Equations, Phys Rev., 4, 345, 1914.

Boldyrev, S.: Spectrum of Magnetohydrodynamic turbulence, Phys. Rev. Lett., 96, 11 5002, 2006.

Bruno, R. and Carbone, V.: The Solar Wind as a Turbulence Laboratory, Living Reviews in Solar Physics, 4, http://www. livingreviews.org/lrsp-2005-4, 2005.

Burlaga, L. F.: Lognormal and multifractal distributions of the heliospheric magnetic field, J. Geophys. Res., 106, 15 917-15 927, 2001.

Carbone, V.: Cascade model for intermittency in fully developed magnetohydrodynamic turbulence, Phys. Rev. Lett., 71, 1546, 1993.

Carbone, V., Veltri, P., and Bruno, R.: Experimental evidence for differences in the extended self similarity scaling laws between fluid and magnetohydrodynamic turbulent flows, Phys. Rev, Lett., 75, 3110, 1995.

Chapman, S. C., Hnat, B., Rowlands G., and Watkins, N. W.: Scaling collapse and structure functions: identifying self-affinity in finite length time series, Nonlin. Processes Geophys., 12, 767-
774, 2005,

http://www.nonlin-processes-geophys.net/12/767/2005/.

Chapman, S. C. and Hnat, B.: Quantifying scaling in the anisotropic turbulent solar wind, Geophys. Rev. Lett., 34, L17103, doi:10.1029/2007GL030518, 2007.

Dendy, R. O., Chapman, S. C., and Paczuski, M.: Fusion, space, and solar plasmas as complex systems, Plasma Phys. Cont. Fusion, 49, A95, 2007.

Frisch U.: Turbulence. The legacy of A.N. Kolmogorov, p. 136, Cambridge University Press, Cambridge, 1995.

Galtier, S., Nazarenko, S. V., Newell, A. C., and Pouquet, A.: Anisotropic turbulence of shear Alfven waves, J. Plasma Phys.,63, 447, 2000.

Giacalone, J., Jokipii, J. R. and Matthaeus, W. H.: Structure of the turbulent interplanetary magnetic field, Ap. J., 641, L61, 2006.

Goldstein, M. L. and Roberts, D. A.: Magnetohydrodynamic Turbulence in the solar wind, Phys. Plasmas, 6, 4154-4160, 1999.

Goldstein, M. L.: Major unsolved problems in space plasma physics, Astrophys. Space Sci.,277, 349, 2001.

Goldreich, P. and Sridhar, S.: Magnetohydrodynamic turbulence revisited, Ap. J,485, 680, 1997.

Hnat, B., Chapman, S. C., Rowlands, G., Watkins, N. W., and Farrell, W. M.: Finite size scaling in the solar wind magnetic field energy density as seen by WIND, Geophys. Res. Lett., 29, 86, 2002.

Horbury, T. S., Forman, M. A., and Oughton, S.: Spacecraft observations of solar wind turbulence: an overview, Plasma Phys. Cont. Fusion,47, B703, 2005.

Hnat, B., Chapman, S. C., and Rowlands, G.: Intermittency, scaling, and the Fokker-Planck approach to fluctuations of the solar wind bulk plasma parameters as seen by the WIND spacecraft, Phys. Rev. E 67, 056404, 2003.

Hnat, B., Chapman, S. C., and Rowlands, G.: Compressibility in Solar Wind Plasma Turbulence, Phys. Rev. Lett. 94, 204502, 2005.

Hnat, B., Chapman, S. C., and Rowlands, G.: Scaling and a FokkerPlanck model for fluctuations in geomagnetic indices and comparison with solar wind epsilon as seen by WIND and ACE., J. Geophys. Res., 110, A08206, doi:10.1029/2004JA010824, 2005.

Hnat, B., Chapman, S. C., Kiyani, K., Rowlands, G., and Watkins, N. W.: On the fractal nature of the magnetic field energy density in the solar wind, Geophys. Res. Lett., 34, L15108, doi:10.1029/2007GL029531, 2007.

Horbury T. S. and Balogh, A.: Structure function measurements of the intermittent MHD turbulent cascade, Nonlin. Processes Geophys., 4, 185-199, 1997.

Hughes, D., Paczuski, M., Dendy, R. O., Helander, P., and McClements, K. G.: Solar Flares as Cascades of Reconnecting Magnetic Loops Phys. Rev. Lett.,90, 131101, 2003.

Iroshnikov, P. S.: Turbulence of a conducting fluid in a strong magnetic field, Sov. Astron., 7, 566, 1964.

Katul, G. G., Albertson, J. D., Chu, C. R., and Parlange, M. B.: Intermittency in Atmospheric Surface Layer Turbulence: The Orthonormal Wavelet Representation, Wavelets in Geophysics, 81, 1997.

Kiyani, K., Chapman, S. C., and Hnat, B.: Extracting the scaling exponents of a self-affine, non-Gaussian process from a finitelength time series, Phys. Rev. E, 74, 051122, 2006.

Kiyani, K., Chapman, S. C., Hnat, B., and Nicol, R. M.: Self- sim- 
ilar signature of the active solar corona within the inertial range of solar wind turbulence,Phys. Rev. Lett., 98, 211101, 2007.

Klimchuk, J. A., and Porter, L. J.: Scaling of heating rates in solar coronal loops, Nature, 377, 131, 1995.

Kolmogorov, A. N.: Local structure of turbulence in an incompressible viscous fluid at very high Reynolds numbers, C. R. Acad. Sci., 30, 301, 1941.

Kraichnan, R. H.: Inertial range spectrum of hydromagetic turbulence, Phys. Fluids, 8, 1385, 1965.

Lu, E. T. and Hamilton, R. J.: Avalanches and the distribution of solar flares, Astrophys. J., 380, L89, 1991.

Matthaeus, W. H. and Goldstein, M. L.: Low-Frequency 1/f Noise in the Interplanetary Magnetic Field, Phys. Rev. Lett. 57, 495498, 1986.

Matthaeus, W. H., Goldstein, M. L., and Roberts, D. A.: Evidence for the presence of quasi- two dimesional nearly incompressible fluctuations in the solar wind, J. Geophys. Res., 95, 20673, 1990.

Matthaeus, W. H., Dasso, S., Weygand, J. M., Milano, L. J., Smith, C. W., and Kivelson, M. G.: Spatial correlation of solar wind turbulence from two point measurements, Phys. Rev. Lett., 95, 231101, 2005.

Merrifield, J. A., Arber, T. D., Chapman, S. C., and Dendy, R. O.: The scaling properties of two dimensional compressible magnetohydrodynamic turbulence, Phys. Plasmas, 13, 012305, 2006.

Merrifield, J. A., Chapman, S. C., and Dendy, R. O.: Intermittency, dissipation and scaling in two dimensional magnetohydrodynamic turbulence, Phys. Plasmas, 14, 012301, 2007.

Milano, L. J., Dasso, S., Matthaeus, W. H., Smith, C. W.: Spectral distribution of the cross helicity in the solar wind, Phys. Rev. Lett., 93, 155005, 2004.

Milovanov, A. V. and Zelenyi, L. M.: Fracton excitations as a driving mechanism for the self-organized dynamical structuring in the solar wind, Astrophys. Space Sci., 264, 317-345, 1998.

Müller, W. -C. and Grappin, R.: Spectral energy dynamics in Magnetohydrodynamic turbulence, Phys. Rev. Lett., 95, 114502, 2005.

Müller, W.-C. and Biskamp, D.: Scaling Properties of ThreeDimensional Magnetohydrodynamic Turbulence Phys. Rev. Lett., 84, 475, 2000.
Politano, H. and Pouquet, A.: Model of intermittency in magnetohydrodynamic turbulence, Phys. Rev. E, 52, 636, 1995.

Politano, H. and Pouquet, A.: von KármánHowarth equation for magnetohydrodynamics and its consequences on third-order longitudinal structure and correlation functions, Phys. Rev. E, 57(R21), 1998.

Schrijver, C. J., Title, A. M., Harvey, K. L., Sheeley Jr., N. R., Wang, Y.-M., van den Oord, G. H. J., Shine, R. A., Tarbell, T. D., and Hurlburt, N. E.: Large-scale coronal heating by the smallscale magnetic field of the Sun, Nature, 94, 152, 1998.

She, Z.-S. and Leveque, E.: Universal scaling laws in fully developed turbulence, Phys. Rev. Lett., 72, 336, 1994.

Sethna, J. P., Dahmen, K. A., Myers, C. R.: Crackling noise, Nature, 410, 242, 2001.

Sorriso-Valvo, L., Carbone, V., Veltri, P., Consolini, G., and Bruno, R.: Intermittency in the solar wind turbulence through probability distribution finctions of fluctuations, Geophys. Res. Lett., 26, $1801,1999$.

Sornette, D.: Critical Phenomena in Natural Sciences, Springer, 2004.

Spangler, S. R. and Spiller, L. G.: An empirical investigation of compressibility in magnetohydrodynamic turbulence, Phys. Plasmas, 11, 1969, 2004.

Taylor G. I.: The spectrum of Turbulence, Proc. R. Soc. A 164, 476, 1938.

Tu, C.-Y. and Marsch, E.: MHD Structures, Waves and Turbulence in the Solar Wind: Observations and Theories, Space Sci. Rev.73, 1-210, 1995.

Tu, C.-Y., Zhou, C., Marsch, E., Xia, L.-D., Zhao, L., Wang, J.-X., and Wilhelm, K.: Solar Wind Origin in Coronal Funnels Science, 308, 519, 2005.

Veltri, P.: MHD turbulence in the solar wind: self- similarity, intermittency and coherent structures, Plasma Phys. Cont. Fusion, 41, A787, 1999.

Watkins, N. W., Credgington, D., Hnat, B., Chapman, S. C., Freeman, M. P., and Greenhough, J.: Towards Synthesis of Solar Wind and Geomagnetic Scaling Exponents: A Fractional Lévy Motion Model, Space Sci. Rev. 121, 271-284, doi:10.1007/s11214-006-4578-2, 2005. 